\title{
EEG Artifacts Reduction by Multivariate Empirical Mode Decomposition and Multiscale Entropy for Monitoring Depth of Anaesthesia during Surgery
}

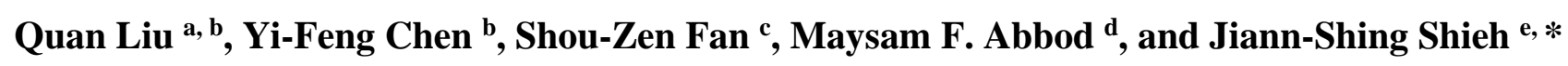

a Key Laboratory of Fiber Optic Sensing Technology and Information Processing, Ministry of Education, Wuhan University of Technology, Wuhan, Hubei 430070 China (e-mail: quanliu@whut.edu.cn)

b School of Information Engineering, Wuhan University of Technology, Wuhan, Hubei 430070 China (e-mail: yifengchen@whut.edu.cn)

c Department of Anesthesiology, College of Medicine, National Taiwan University, Taipei 100 Taiwan (e-mail: shouzen@gmail.com)

d Department of Electronic and Computer Engineering, Brunel University London, Uxbridge, UB8 3PH, UK (e-mail: maysam.abbod@brunel.ac.uk)

e Department of Mechanical Engineering, and Innovation Center for Big Data and Digital Convergence, Yuan Ze University, Taoyuan, Chung-Li 32003 Taiwan. (e-mail: jsshieh@saturn.yzu.edu.tw)

* Author to whom correspondence should be addressed; E-Mail: jsshieh@saturn.yzu.edu.tw; Tel.: +886-3-463-8800 (ext. 2470); Fax: +886-3-455-8013.

The total number of words of the manuscript: 7685

The number of words of the abstract: 215

The number of figures: 8

The number of tables: 3

Abstract: Electroencephalography (EEG) has been widely utilized to measure the depth of anaesthesia (DOA) during operation. However, the EEG signals are usually contaminated by artifacts which have a consequence on the measured DOA accuracy. In this study, an effective and useful filtering algorithm based on multivariate empirical mode decomposition (MEMD) and multiscale entropy (MSE) is proposed to measure DOA. Mean entropy of MSE is used as an index to find artifacts-free intrinsic mode functions (IMFs). The effect of different levels of artifacts on the performances of the proposed filtering is analyzed using simulated data. Furthermore, 21 patients' EEG signals are collected and analyzed using sample entropy to calculate the complexity for monitoring DOA. The correlation coefficients of entropy and bispectral index (BIS) results show $0.14 \pm 0.30$ and $0.63 \pm 0.09$ before and after filtering respectively. Artificial neural network (ANN) model is used for range mapping in order to correlate the measurements with BIS. The ANN method results show strong correlation coefficient $(0.75 \pm 0.08)$. The results in this paper verify that entropy values and BIS have a strong correlation for the purpose of DOA monitoing and the proposed filtering method can effectively filter artifacts from EEG signals. The proposed method performs better than the commonly used wavelet denoising method. This study provides a fully adaptive and automated filter for EEG to measure DOA more accuracy and thus reduce risk 
related to maintenance of anaesthetic agents.

Keywords: Depth of anaesthesia, Electroencephalography, Multivariate empirical mode decomposition, Filtering, Multiscale entropy, Mean entropy value

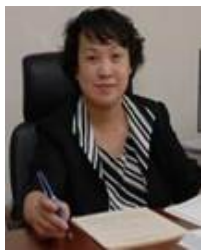

Quan Liu received Ph.D. degree from the Wuhan University of Technology, China, in 2003. Currently, she is a Professor at the Wuhan University of Technology and the Council Member of Chinese Association of Electromagnetic Compatibility. Her research interests include signal processing, robot hardware and electronics, embedded systems and applications.



Yi-Feng Chen received M.Sc. degree in the School of Information Engineering from Wuhan University of Technology, China, in 2014. Currently, he is a Ph.D. candidate. His research area is in biomedical signal processing, especially in brain monitoring during anaesthesia and the Intensive Care Unit.

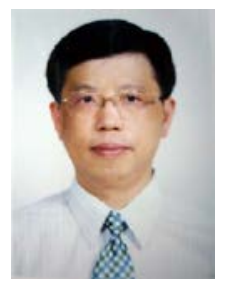

Shou-Zen Fan received Ph.D. degree from the National Taiwan University in 1994. Currently, he is an Associate Professor at National Taiwan University. His research interests include pediatric anaesthesia and pain management, airway management, anaesthesia for liver transplantation, and anaesthetic automatic control and expert systems.

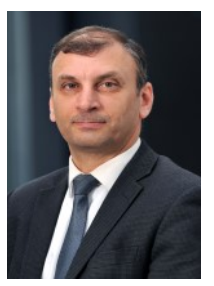

Maysam F. Abbod received the Ph.D. degree in Control Engineering from the University of Sheffield, U.K., in 1992. Currently, he is a Reader of Electronic Systems in the Department of Electronic and Computer Engineering, Brunel University London, UK. His main research interests are in intelligent systems for modelling, control and optimisation.

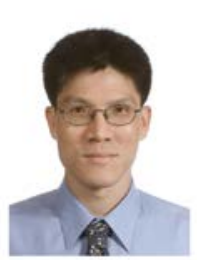

Jiann-Shing Shieh received Ph.D. degree in Automatic Control and Systems Engineering from the University of Sheffield, U.K., in 1995. Currently, he is a Professor at the Yuan Ze University. His research interests include intelligent analysis and control, bio-signal processing, critical care medicine monitoring and control, medical automation, and brain death index research. 


\section{Introduction}

General anaesthesia (GA) is usually administered to safely induce a reversible loss of consciousness allowing the performance of complex surgical and diagnostic procedures under optimal conditions [1]. It is an essential procedure for patients to avoid unbearable pain and unpleasant memories during surgical operations. However, how anaesthetic drugs are induced and maintain the behavioural states of general anaesthesia is an important question in medicine and neuroscience. Mashour [2] interpreted the anaesthesia by utilizing the concepts of cognitive binding and unbinding. The cognitive unbinding of gamma activity is a mechanism of anaesthetic activity. However, the proposed paradigm cannot accounts for immobility mediated in the spinal cord. Recent works explained the molecular and neuronal mechanisms for general anaesthetics [3, 4]. The anaesthetic targets, such as $\gamma$-aminobutyric acid type A (GABAA) receptors, $\mathrm{N}$-methyl-d-aspartate (NMDA) receptors and two-pore-domain $\mathrm{K}^{+}$ channels play a crucial role in anaesthetic-induced loss of consciousness. Nevertheless, how the relevant neuronal pathways are affected by general anaesthetics remains to be understood more clearly. Since general anaesthetics mechanism is not well understood, methodologies for assessment of depth of anaesthesia (DOA) are controversial. Kenwright et al. [5] developed a DOA monitor based on cardiovascular signals. However, because general anaesthesia involves loss of consciousness which is more related to brain activities, objective measures of depth of anaesthesia have been focused mostly on EEG and EEG-derived measurements.

Electroencephalogram (EEG) has been widely used in clinical environment for the diagnosis of various diseases related to brain system [6-8]. Especially, researchers have introduced many kinds of monitors to quantify anaesthesia awareness level based on EEG analysis during surgery, such as the bispectral index (BIS) [9], EEG entropy monitoring developed by Datex-Ohmeda [10], and the Narcotrend monitor [11] during general anaesthesia. The electrical activity of the cerebral cortex conducts the EEG signal, so the features of EEG change with the patient's brain states [12]. The brain is a complex dynamical systems existing functional connectivity with other physiological oscillations [13]. Tomislav Stankovski et al. [14] introduced a method to detect synchronization and determine the coupling functions in time-variable coupled dynamics. Furthermore, a method detecting the effective phase connectivity based on dynamical Bayesian inference is also proposed to analyse multivariate networks of oscillators [15]. General anaesthesia thus can be assessed by cross-frequency couplings of combined dynamics between neuronal, cardiac and respiratory oscillations [13].

However, the activities originating from other sources rather than the brain are also recorded by EEG. These recorded signals with non-cerebral origin are called artifacts. Unlike noise defined by unwanted interference arising from unknown origin [16], an artefact is an unwanted activity but from known deterministic origin. For example, the electrooculography (EOG) is generated by the movement of eyes during blinking and electromyography (EMG) is the muscle activation. Generally, the brain activities signal are very weak, and the EEG signals are prone to be corrupted by artifacts like EOG and EMG. The artifacts in EEG signal increase the difficulty of monitoring DOA in clinical surgeries.

There is a need to remove the artifacts prior to analysis of the EEG and its use in DOA monitor. Various methods have been used to deal with EEG artifacts. Basically, linear methods based on Fast Fourier Transform (FFT) in frequency domain are widely used to remove unwanted signals [17]. Although it is easy to design and implement, the linear methods are suitable for linear and stationary signals. However, the EEG is non-stationary and non-linear. So these methods are not so effective for 
EEG signals. To solve these problems, non-linear filtering methods based on wavelet transform (WT) have been proposed [18-20], which has been applied for the elimination of different types of artifacts [21] and for removal of some electrophysiological artifacts, such as ocular movement [22], and heart signal [23] from the EEG signals. However, wavelet approaches are limited to its basis functions which are fixed, and so lead to the fail to satisfy all real signals, especially non-stationary and nonlinear signals. Another approach to artifact detection involves the use of independent component analysis (ICA) [24]. It is a special case of blind source separation (BSS). ICA separates a multivariate signal into a set of statistically independent components (IC). The ICs related to artifacts can be removed. EOG artifact removal on the basis of isolation into independent components (ICs) using BSS has been demonstrated in [25]. However, this method cannot separate a single-channel signal. Currently, a nonlinear mode decomposition (NMD) algorithm based on the wavelet transform (WT) was introduced as a class of adaptive decomposition tool [26]. It separates a time series into several oscillations with physically meaning. Similarly, empirical mode decomposition (EMD) is developed as an important algorithm to analyze non-stationary and non-linear signals [27]. Rehman and Mandic [28] extended EMD to multichannel and introduced multivariate empirical mode decomposition (MEMD). In contrast to the classical Fourier and Wavelet decomposition with fixed basis functions, the EMD and MEMD methods can separate signals into a set of intrinsic mode functions (IMFs) adaptively and do not need predefined basis functions. EMD is wildly used to filter artifacts from physiological signals. An EMD-based filter was used to remove muscle artifact from EEG data in [29]. The EOG artefact was also removed from the EEG by EMD-based approach in [30].

Nevertheless, when the main pure signal is reconstructed from IMFs, the main problem is how to effectively determine IMFs related to the useful components. IMFs are selected all based on the empirically determined findings artificially in the convention method [31, 32]. However, if the recordings are very long, the process of manual artifact rejection implies a significant increase in the time needed to fully process a dataset. Additionally, it is impossible that selected IMFs meet various artifacts conditions for clinical applications. In this paper a new index that is mean entropy value of multiscale entropy (MSE) has been found in application of determining information artifacts-free IMFs adaptively.

The MSE proposed by Costa et al. [33, 34] is an improved method from sample entropy [35] used to measure the complexity of signals over different time scales. The MSE method has been developed as measures of DOA for clinical application [36]. It is used to measure the mean entropy value of each extracted IMF and combination signal without using any reference. However, as with many other monitoring DOA methods, MSE is also exposed to artifacts in the EEG. In order to understand what the artifacts make effect on MSE of EEG, simulation signals consisting of real EEG and simulation artifacts have been used in this paper. Furthermore, in this paper, the MSE values are calculated from EEG to indicate the DOA of patients compared with a gold standard (e.g. BIS). For the sake of being consistent with BIS values, MSE values are mapped into BIS values range by regression model. In machine learning and related fields, artificial neural network (ANN) is a very popular machine learning model based on statistics. Therefore, ANN is utilized for building the regression model in this study.

\section{Methods}

\subsection{The EEG Database Preparation}


There are two parts of the EEG datasets. One is collected from ten subjects. EEG signals with $64 \mathrm{~s}$ data length are recorded when the subjects have consciousness and close eyes. The signals then pass through a band-pass filter at $0.5-60 \mathrm{~Hz}$ and a north filter at $60 \mathrm{~Hz}$. This part is used as quasiuncorrupted EEG signals. Then the modelled artifacts are added into the uncorrupted EEG to obtain the synthetic data. Another dataset is collected from twenty one subjects under general anaesthesia during the whole surgery. This part is used as the real EEG to evaluate the proposed method in practice application.

\subsubsection{Simulated datasets}

The quasi-uncorrupted EEG signals are obtained via a forehead mounted sensor by MP60 system (Philip, IntelliVue MP60 BIS module). Ten healthy volunteers datasets were collected which compromise of 64 s EEG signals, the subjects were conscious but with their eyes closed.. The subjects were sitting in a comfortable armchair. A plot of the quasi-uncorrupted EEG signals is presented in Fig. 1. The EEG collected during awake state performs more non-stationary and contains more variability than that during the general anaesthesia state. During the resting-state in the absence of external stimulation, noise drives the dynamics of brain networks and increases its variability [37]. So the awake EEG signal has higher complexity, which can be used to indicate the depth of anaesthesia. This study investigates the effect of artifacts on the complexity of EEG signal. The EEG during whole stage of surgery containing the awake and anaesthesia state is tested, while the artifacts are mainly appeared in awake stage, so the EEG collected from this stage is used as the simulated signal by adding modelled artifacts. Furthermore, the common artifacts containing EMG, EMG and baseline drift are excluded from the collected EEG of resting-state, and the $60 \mathrm{~Hz}$ power-line interference is also filtered by a north filter. In this case, the EEG without these four artifacts can be considered as the relatively uncorrupted signal.

In order to evaluate the artifacts reduction performance of the proposed filter algorithm in this study, four different representative artifacts are selected for simulation. The artifacts are EOG, EMG, $60 \mathrm{~Hz}$ power-line interferences and baseline drift, respectively. These four different types of artifacts are added to the uncorrupted EEG signal artificially. All contaminated signals are also reduced to different artifacts levels, ranging from $10 \mathrm{~dB}$ to $-20 \mathrm{~dB}$ with a step of $-1 \mathrm{~dB}$, related to the EEG level. The artifacts simulation algorithm refers to the works in [32, 38]:

1) EOG interference because it is the most common artifact in EEG. the real EOG signals is added to the EEG signal in order to simulate this type of artifact. The EOG used is Dataset 2a of BCI Competition IV [39]. This dataset consists of 3 EOG monopolar channels. Since the second EOG channel can properly represent the vertical EOG, specifically eye blinks that are to be removed from EEG, this channel is used for this work. Illustration of the EOG interference with three levels on an uncorrupted EEG is shown in Fig. 2 (a).

2) EMG interference because of its random properties and high frequency content. Friesen, et al. [38] have modelled EMG using zero-mean band-limited Gaussian noise with the frequency range from dc to $10 \mathrm{kHz}$. Furthermore, Chang [32] has modelled the EMG using a normal distributed random number implemented by 'randn' function in MATLAB. Therefore, the random noise modelled by a random number with normal distribution is added into the EEG to simulate the EMG artefact in this study. The random numbers are generated using the "randn" function in MATLAB. Illustration of the EMG interference with three levels on an uncorrupted EEG is shown in Fig. 2 (b). 
3) Power-line interference because it is ubiquitous. It is modelled by $60 \mathrm{~Hz}$ sinusoidal function. 60 $\mathrm{Hz}$ artifact is generated using the "sin" function in MATLAB. Illustration of the power-line interference with three levels on an uncorrupted EEG is shown in Fig. 2 (c).

4) Baseline drift duo to movement because of its low frequency properties. A low frequency sinusoidal signal is added to the uncorrupted EEG to simulate this kind of artifact. It is modelled by a $0.333 \mathrm{~Hz}$ sinusoidal function and generated using the "sin" function in MATLAB like power-line interference. Illustration of the baseline drift with three levels on an uncorrupted EEG is shown in Fig. 2 (d).

\subsubsection{Real EEG dataset}

The real EEG signals are collected from twenty one patients with general anaesthesia whose ages range from 21 to 75. They have accepted ear, nose and throat (ENT) surgery at the National Taiwan University Hospital (NTUH) of Taiwan. The monitoring EEG device used in the operation room is the Philips IntelliVue MP60. Generally, a whole surgical procedures includes four stages: the preoperation, induction, maintenance and recovery [40]. Patients have complete consciousness during the pre-operation stage, and always blinked eyes, thus the measured EEG signals contained EOG and EMG artifacts as shown in Fig. 3 (a) and (b). During the induction stage and the beginning of maintenance stage, patients needed to be moved by the doctors, especially their head, because of intubation and assisted respiration, so the EEG signals were badly corrupted with baseline drift plotted in Fig. 3 (c). During the final stage, the collected EEG signals contained little artifacts due to the close of the operation. In this study, propofol, sevoflurane and desflurane were used to induce and maintain anaesthesia. These are three commonly used anaesthetics based on the same mechanisms of action via $\gamma$-aminobutyric acid type A (GABAA) receptor [41]. Previous studies have illustrated that propofol is not significantly different from sevoflurane or desflurane for general anaesthesia [42-44].

\subsection{EMD/MEMD Based Filter}

According to EMD technique [27], the complex signal $X(t)$ is first decomposed into a set of limited simple functions called intrinsic mode function (IMF) through the sifting process, with each one having a distinct time scale. The frequencies of IMFs range from high to low in each level. Each IMF obtained by EMD represents a band-limited part of original signal [45]. Different from most decomposition algorithms with a fixed base, this decomposition method adapt to the local characteristic of data and without priori. In order to reconstruct the original signal $X(t)$, all the IMFs decomposed by EMD are summed up and the final residue is expressed as follows:

$$
X(t)=\sum_{i=1}^{n} c_{i}(t)+r_{n}(t)
$$

where $X(t)$ is the original data; $n$ is the total number of IMFs; $c_{i}$ is the $i$ th IMFs; and $r_{n}$ is the $n$th residue.

The MEMD was proposed as an extended EMD method in order to make EMD suitable for processing of multichannel signals by Rehman and Mandic in 2010 [28]. MEMD decomposes an input multivariate signal $\mathbf{X}(t)=\left\{x_{1}(t), x_{2}(t), \cdots, x_{n}(t)\right\}$ into a multidimensional set of IMFs. To achieve that, input signal projections are taken directly along different directions in n-dimensional spaces to calculate the local mean. This step is necessary since calculation of the local mean, a crucial step in EMD algorithm, is difficult to perform due to the lack of formal definition of maxima and minima in higher dimensional domains. In order to obtain projections of the input signal in n-dimensional spaces, the 
sampling scheme based on low discrepancy Hammersley sequence was used. Once the projections along different directions in multidimensional spaces are obtained, their extrema are interpolated via cubic spline interpolation to obtain multiple signal envelopes; these envelopes are then averaged to obtain the local mean of a multivariate signal. Then the multivariate IMFs are extracted from the input signal by the sifting process similar to EMD process. However, unlike EMD, MEMD better aligns the corresponding IMFs from different channels across the same frequency range. When adding extra channels containing white noise into the input signal, MEMD resolves the mode mixing problem in the existing EMD algorithm [46].

The IMFs decomposed by EMD/MEMD represent specific components with band-limited frequency of original signal, so the artifacts can be filtered from signals by summing partial IMFs according to the following equation:

$$
\hat{X}(t)=\sum_{\mathrm{p}}^{q} c_{i}(t)
$$

where $\hat{X}(t)$ is the filtered signal; $c_{i}$ is the ith IMFs decomposed by EMD/MEMD; assuming $N$ is the total number of IMFs then $p, q \in[1, N]$. When $p=1, q=N$, that becomes equivalent to the original signal $X(t)$. When $1<p<q=N$ that means a low pass filter; when $1=p<q<N$ that represents a high pass filter; when $1<p<q<N$ that indicates a band pass filter, and when $1<q<p<N$, (2) can be expressed as in (3) which presents a band stop filter.

$$
\hat{X}(t)=\sum_{1}^{q} c_{i}(t)+\sum_{\mathrm{p}}^{N} c_{i}(t)
$$

EMD/MEMD behaves as a filter bank without a predefined cut-off frequency [47] and can be seen as a set of adaptive filters [48, 49].

\subsection{Sample Entropy and Multiscale Entropy}

Sample entropy (SampEn) developed by Richman and Moorman [35] is an algorithm to estimate the complexity of signal. It is widely applied to many fields to analyze the complexity of time series data, including physiological data such as EEG data and heart rate variability [50, 51]. Mathematically, for a given embedding dimension $m$, tolerance $r$ and a time series $\{x(\mathrm{n})\}=x(1), x(2), \ldots, x(N)$ with $N$ data points, SampEn can be calculated as follows:

1) Construct template vectors of length $m$, defined as

$$
X_{m}(i)=\left\{x_{i+1}, x_{i+2}, x_{i+3}, \ldots, x_{i+m-1}\right\}, 1 \leq i \leq N-m+1
$$

2) Define the distance function between vectors $X_{m}(i)$ and $X_{m}(j)$ to be the Chebyshev distance, denoted by $d\left[X_{m}(i), X_{m}(j)\right]$, as

$$
d\left[X_{m}(i), X_{m}(j)\right]=\max \left(\left|X_{m}(i+k)-X_{m}(j+k)\right|\right), 0 \leq k \leq m-1 \text { and } i \neq j
$$

3) Given a vector $X_{m}(i)$, count the number of vector $X_{m}(j)$ subjecting to the criterion of similarity $d\left[X_{m}(i), X_{m}(j)\right] \leqslant r$ for $1 \leqslant j \leqslant N-m$ to exclude self-matches denoted as $B_{i} \cdot B_{i}^{m}(r)$ is defined as:

$$
B_{i}^{m}(r)=\frac{1}{N-m-1} B_{i}
$$

4) For $1 \leqslant i \leqslant N-m, B_{i}^{m}(r)$ is defined as follows:

$$
B^{m}(r)=\frac{1}{N-m} \sum_{i=1}^{N-m} B_{i}^{m}(r)
$$

5) Similarly, set $m=m+1$ and repeat steps 1 ) to 4$)$, where $A_{i}^{m}(r)$ is defined as

$$
A^{m}(r)=\frac{1}{N-m} \sum_{i=1}^{N-m} A_{i}^{m}(r)
$$

6) Define the sample entropy to be 


$$
\operatorname{SampEn}(m, r, N)=-\ln \frac{A^{m}(r)}{B^{m}(r)}
$$

Generally, the value of $m$ is set to be 2 and the value of $r$ to be $0.2 \times s t d$, where std stands for the standard deviation of the data.

Multiscale entropy (MSE) technique is proposed as a method considering the multiple time scales in a data sequence [33, 34]. The MSE can be calculated as follows:

1) Given a time series $\{\mathrm{x}(\mathrm{n})\}=\mathrm{x}(1), \mathrm{x}(2), \ldots, \mathrm{x}(N)$ with $N$ data points, multiple new time series can be obtained by averaging the data points located in a window with fixed length which slides with the step $\tau$. The new time series are called coarse-grained time series defined as $y_{j}^{(\tau)}$ and calculated according to equation (10):

$$
y_{j}^{(\tau)}=\frac{1}{\tau} \sum_{i=(j-1) \tau+1}^{j \tau} X_{i}
$$

where $\tau$ is the scale factor and $1 \leqslant j \leqslant N / \tau$.

2) Calculate the complexity of each coarse-grained time series $\left\{y^{\tau}\right\}$ using SampEn algorithm, then for each scale factor, there are a corresponding value.

\subsection{Definition of Mean Entropy Value}

The SampEn definition mentioned above is a special case of MSE scale with $\tau=1$, therefore entropy value containing SampEn and MSE can be universally defined as a function of $\tau$. According to the previous studies [33, 34],the results confirm that $1 / \mathrm{f}$ noise is more complex compared with white noise. In fact, $1 / \mathrm{f}$ noise contains more information over times scales than white noise. Due to the conclusion drawn from above, the complexity of signal is not only decided by single scale. Therefore, a new index called mean entropy value (MEV) is defined in equation (11). The MEV is mean entropy value based on MSE with all scale factors to represent overall complexity of signal.

$$
M E V=\frac{1}{M} \sum_{\tau=1}^{M} M S E_{\tau}
$$

where $M$ is the total scale number and $M S E_{\tau}$ is the MSE value at scale factor $\tau$.

It is true fact that different types of artifacts have different effect on the complexity of EEG. In addition, it is not clear how the artifacts affect the complexity of EEG signal, i.g. do artifacts decreases or increases the complexity and which scale entropy value of MSE is effected. Aim to investigate the relation mentioned above, 10 cases' EEG signals are used, and then four types of artifacts are separately added into the original EEG as the simulation signal. The EEG signals and simulated artifacts have been explained above. The results of different amounts of artifact on one case's MSE curves are shown in Fig. 4 (a), (b), (c) and (d) respectively. Fig. 4 (a) and (d) show three different types of behaviours: (1) For a special artifact level, the entropy measure for both uncorrupted and corrupted EEG increases on small time scales and then stabilizes to a constant value. (2) For a special scale, entropy value decreases on all time scales as the artifact level increasing. (3) For all scales, the complexity of uncorrupted EEG is higher than corrupted EEG based on entropy value. Fig. 4 (b) shows the results of the MSE analysis of EEG corrupted with EMG artifact. For some artifact level such as more than $9 \mathrm{~dB}$ containing the original EEG, the entropy measured from the EEG increases on small time scales and then stabilizes to a constant value, when the artifact level is from 3 to $8 \mathrm{~dB}$, the entropy value apparently decreases on small time scales and then gradually increases, and 
maintains a constant value in the final. When the artifact level increases, the entropy decreases monotonically, similar to white noise. On large time scales, the entropy decreases monotonically. It indicates that the coarse-grained time series become generally more regular with the increasing of scale factor. For a special scale, when signal to noise ratio is more than $3 \mathrm{~dB}$, the entropy value increases on time scale 1 and 2 as the artifact level increasing. When signal to noise ratio is $3 \mathrm{~dB}$, the entropy value were basically identical on all artifact levels. When signal to noise ratio is less $3 \mathrm{~dB}$, the entropy value decreases. Therefore, the complexity of EEG signal corrupted with EMG artifact cannot be determined by some specific time scales. In Fig. 4 (c), the results of the MSE analysis of EEG which is corrupted with power-line artifact are presented. The entropy value markedly increases on small artifact level and then gradually increases on scale 1 and 2. When scale is more than 3, the entropy value decreases like the other three situations. The complexity of EEG signal corrupted with power-line artifact also cannot be confirmed by some specific time scales.

Based on the above analysis, the entropy value of EEG corrupted with low frequency artifact such as baseline drift and EOG decreases with the artifact level increasing over all time scale. These artifacts can reduce the complexity of EEG signal. Nevertheless, broadband artifact (e.g. EMG) and high frequency artifact (e.g. power-line) increase the complexity on small time scales and decrease complexity on large time scales. Therefore, in order to better study the complexity of an EEG signal, not only the specific entropy is needed to be considered, but also the overall features across multiple scale factors.

Next, to assess the effects of artifact level, the MEV of the EEG time series corrupted is compared with different amount of simulated artifact from 10 subjects. This way, the mean and standard deviation of MEV (error bars) related to the artifact level of the simulated series are shown in Fig. 5 (a), (b), (c) and (d), respectively. It is fond that for all time scales, a highest value of MEV is assigned to original EEG without artifact, and the MEV monotonically decreases with artifact level increasing.

\subsection{Adaptive IMFs Selection}

The proposed algorithm relies on the basic fact that the uncorrupted EEG signals have maximum complexity based on the above conclusions. The artifact components decrease the MEV of EEG. Therefore, in the whole procedure, IMFs are selected adaptively to maximize the complexity of reconstructed signal. The IMF with maximal MEV is considered as a basic component decomposed from EEG. Then other IMFs are added to the basic component to estimate complexity trend of reconstructed signal. If the complexity increases, the corresponding IMFs are accepted as components to construct EEG signal and the IMFs are discarded as artifact the other way round. In this way, there is no need to traverse all the possible combinations of different IMFs to achieve the goals. Details of the proposed method are outlined as follows:

1) Decompose the multivariate signal constituted by one channel original EEG and two extra channel white noise through MEMD.

2) Reserve the IMFs related to EEG signal, the total number of IMFs is denoted by $M$.

3) Calculate MEV for each reserved IMFs by applying MSE and find the maximal MEV denoted by $M E V_{\max }$, the corresponding IMF is $I M F_{i}$ and IMF index is $i$.

4) Reconstruct signal $S$ by adding $I M F_{i}$ and one of the remaining IMFs as $\left\{S_{1}, S_{2}, S_{3}, \ldots, S_{M-1}\right\}$ according to equation (12)

$$
S=I M F_{i}+I M F_{j}, \text { for } 1 \leq \mathrm{j} \leq M, j \neq i
$$


5) Calculate MEV for each of reconstructed signal $\left\{S_{1}, S_{2}, S_{3}, \ldots, S_{M-1}\right\}$, as $\left\{M E V_{1}, M E V_{2}, M E V_{3}, \ldots, M E V_{M-1}\right\}$.

6) Compare each of $\left\{M E V_{1}, M E V_{2}, M E V_{3}, \ldots, M E V_{M-1}\right\}$ with $M E V_{\max }$, if $M E V_{k}>M E V_{\max }$ for $1 \leq k \leq M-1$, mark the corresponding IMF of $M E V_{k}$ which is considered as clean signal without artifact.

7) Reconstruct the filtered EEG signal by summing all of the marked IMFs from step 6) and $I M F_{i}$.

\section{Results}

\subsection{Simulation Results}

In our simulation, the uncorrupted EEG signals illustrated in section 2.1.1 are employed for analyzing and de-noising signals. Then different level artifacts are added to the uncorrupted EEG signal to evaluate our proposed method in terms of signal to noise ratio (SNR). More specifically, the adopted performance measure is the difference between the SNR after and before de-noising. The detailed SNR performances are shown in Table 1 and Fig. 6. The results of SNR improvements after filtering increase with input SNR level in common with EOG artifact. It means that the performance of proposed filter method is more effective in low SNR signals. The reason of this result is that MEMD cannot decompose the EEG signals with weak artifact into artifact-dominated IMFs and signaldominated IMFs completely, therefore it cannot separate the artifact and signal from the signals corrupted with artifacts, the removed IMFs are mixture of artifact and signal, so while the proposed method can reduce artifact, it may also remove the signal information. On the other hand, as the artifact level decreases (SNR increases), the complexity of EEG corrupted with artifacts is closer to uncorrupted signals. It is also difficult to decide which IMFs are signal dominated. As indicated in Table 1, the de-noising method doesn't work when SNR before de-noising is more than 3dB for EOG artifact. The signals with other three types of artifacts are also experiencing a similar situation. Generally, one of the mainly characteristics of EEG is the signal is very weak. The amplitude is about from $10 \mu \mathrm{V}$ to $100 \mu \mathrm{V}$ for an adult [52]. However, the amplitude of artifacts can be quite large relative to the EEG signals of interest. So the SNR is very poor. The proposed method is highly suitable for use to remove artifact from EEG.

Globally, when comparing the performance for EOG artifact, EMG artifact, power-line and baseline drift, as shown in the last line of Table 1, there is a significant difference and the method is far more effective in reducing artifact for power-line and baseline drift. Because power-line and baseline drift are modelled by sinusoidal function with a single frequency band. The corresponding artifact components are located within just one IMF. Sinusoidal functions are perfect regularity so the effect of power-line and baseline drift is more significant on EEG signal. The MEV index can more accurately discriminate between artifact-dominated and signal-dominated IMFs.

\subsection{Real EEG Signal Processing via Proposed Method}

When monitoring EEG signal from patients during surgery in operation rooms, artifacts is probably inevitable. Therefore, in order to measure DOA correctly, the artifacts must be removed as far as possible. In this study, the proposed method is used to filter the real EEG signal collected from patients at the National Taiwan University Hospital (NTUH) of Taiwan. Then the MSE algorithm is applied to monitor the DOA instead of BIS recorded in real time during the surgery through MP60 system. Because the essence of BIS is to take the EEG signal, analyze it, and process the result into a single number every five seconds, in order to consistent with BIS recordings, a sliding window using fixed- 
size of 5 seconds including 625 points is needed. The window moves once every five seconds for real time DOA monitoring. However, it is possible that the length of MSE results is not equal to BIS, spline interpolation is used to fill the gaps in the results. The previous study has certified that in the large scales with the coarse-graining procedure MSE only relates to the complexity of low frequency components of EEG. In practice, however, there can be an increase in high frequencies when people awake. It has no advantage monitor the DOA of patients undergoing operations at large scales. So the scale factor used in research is set to be 1 to monitor the DOA during the whole operation. Furthermore, in order to illustrate the performance of the proposed filter method, a comparison is made with the commonly used wavelet denoising method. The soft heuristic Stein's unbiased risk estimate (SURE) thresholding are applied to the detail coefficients from 1 to 6 and wavelet Daubechies 4 (db4) is selected as the basis function.

One case EEG recording is given as an example to illustrate the relationship between MSE and BIS in monitoring DOA during the whole operation. The EEG data is collected from a female patient, aged 35, during a laparoscopic (LSC) operation. The whole surgery time is divided into four stages: preoperation, induction, maintenance and recovery as shown in Fig. 7. It is revealed from Fig. 7(c) and (d) that the entropy values vary from high to low and back to high exactly similar to BIS values during the whole surgery. The detailed results listed in Table 2 provide statistics for the entropy measurement for each stage during the whole surgery. As discussed above, the mean values of entropy after filtering for each patient vary from high to low and back to high consistent with BIS. Especially, the mean value $(1.65 \pm 0.20)$ of stage 1 after filtering is much higher than the value $(0.85 \pm 0.26)$ before filtering. The reason of this problem is that patients have consciousness and blink or move constantly during preoperation stage. Thus the recorded EEG signals are seriously disturbed by interferences which reduce the complexity of corresponding signals. Obviously, the correlation coefficients are higher after filtering compared with before during pre-operation, induction and recovery because of the artifacts removing. The patients have no consciousness during maintenance stage, original signals are relatively clean, and so there are no significant differences between original and reconstructed EEG. The relationship between the entropy and BIS is linear $(\mathrm{p}<0.01)$ for each stages after filtering.

Moreover, to illustrate the corresponding performance for the proposed filtering approach, the correlation coefficients between MSE and BIS during the whole operation in twenty one surgeries are calculated. The results are shown in Table 3 as the measures of efficiency of artifact reduction and expressed as mean \pm standard deviation. It is suggested that there is a very small increase from $0.14 \pm 0.30$ to $0.15 \pm 0.27$ after using wavelet denoising method, while a strong linear relationship is present between entropy values after using proposed filter method and BIS values increasing from $0.14 \pm 0.30$ to $0.63 \pm 0.09$. Thus the interferences or artifacts contained in EEG signals are filtered effectively by the proposed method and it performs better than wavelet method. Furthermore, the entropy and BIS values are regarded as dependent variables and independent variables to train the ANN model in application of regression. According to the results as shown in $5^{\text {th }}$ and $6^{\text {th }}$ columns in Table 3, there is a stronger linear relationship between the two variables than that without ANN, and it is evident that the degree of correlation are much stronger in comparison with the result after filtering in $3^{\text {rd }}$ and $4^{\text {th }}$ columns. Furthermore, the proposed method still performs better than the wavelet denoising algorithm, the mean and standard deviation of coefficients are $0.75 \pm 0.08$ and $0.35 \pm 0.18$ for our proposed method and wavelet method, respectively. 
However, as indicated in Table 3, the strength of the correlations remains weak after filtering for some cases (e.g. case 18 and case 21). The reason is that BIS algorithm sets the values to be -1 when EEG signals are disturbed by interferences or artifacts seriously. At the same time, the proposed filtering method in this paper can remove the artifacts effectively so that entropy values remain the capability to monitor the DOA and patient's states. So the combination of the filtering method and MSE method is superior to BIS module at least in this aspect when EEG signals are contaminated by interferences or artifacts seriously. Fig. 8 shows the EEG signal and corresponding states measured from case 21 during the whole surgery which gives a detailed account of the problem mentioned above. For the positions (2), (3) and (4), the BIS values are -1, but the entropy values are normal. As expected, the original EEG during (2) and (3) are mainly affected by EOG and the operation records of case 21 show that and high-frequency electric knife which can badly affect the EEG measurement is used by doctor during the interval (4).

\section{Discussion}

Artifact cancellation from EEG signals is a long-standing problem for accurate evaluation of DOA. The MEMD as an extended application of EMD performs very useful and powerful to analyze nonlinear and non-stationary signals. The MSE is proposed to evaluate the complexity of time series on multiple time scales. The EEG signals corrupted with artifacts are decomposed into several IMFs by MEMD, by this way the artifacts and EEG are distributed in different IMFs. However, IMFs selection to adaptively decide which IMFs are signal-dominated and which IMFs are artifact-dominated is the major problem. Conventionally, IMFs are decided by observing their spectrum artificially or compared with reference signals. Higher order statistics criteria [53] and thresholding approach [54] inspired from wavelet thresholding methods to evaluate the artifact level of each IMF only can be applied to detect the IMFs contaminated by white Gaussian noise. In practice, it is impossible that selected IMFs meet various artifacts conditions for clinical applications. To solve this problem, a new index named MEV based on MSE is found in application of determining noisy IMFs automatically and adaptively. In analysis of EEG signals through the MSE with the coarse-graining procedure, effects of different amounts of artifacts mentioned in this paper on MSE curves indicate that the complexity of normal EEG signal markedly decreases. If the IMFs contain artifact, the MEV must be lower. This result suggests that the monotonic decrease of MEV maybe related to the relatively low complexity of the artifact. Furthermore, this result further confirms the fact that the original clean EEG signals collected from young healthy subjects make a loss of complexity when corrupted with artifact. Based on this fact, the IMFs is reserved in order to maximize the complexity of the reconstructed EEG signal. Therefore, the proposed method provides a solution to fully online and automated artifact cancellation for DOA monitoring based on EEG signal in operation room and ICU.

The major principle in the whole filtering procedure is to maximize the complexity of reconstructed signal. The IMF with maximal MEV is considered as a reference signal to find other signal-dominated IMFs. In this procedure, it is possible to accept artifact-dominated IMFs or reject signal-dominated IMFs because the reference IMF does not equate to clean original EEG. Under these circumstances, comparing all the possible combinations of IMFs to find the optimal solution maximizing the complexity can be regarded a better method. This method will be tested and compared with the methods proposed in other papers. Finally, the feasibility of the proposed method will be used to characterize other data in the future work. 
In the present work, only four types of artifacts or artifacts have been modelled and analyzed for simulation. In future work, this study will be extended to other artifact models. In addition, the results seen in Table 1 shows that the filtering exhibits poor performance under the circumstance of high SNR. One of the reasons is that MEMD cannot separate the artifact and EEG from the original signals into different IMFs completely, and this may be due to the interpolation algorithm used in the sifting process and thus our team would like to search for other interpolation algorithms other than cubic splines to solve this problem. Furthermore, to confirm the effectiveness of the proposed filtering method, a large more class of experiments under different experimental conditions such as sampling rates, scale number, and sample sizes are needed. In particular, the MSE method takes multiple scales into account for complexity calculation, and the MEV inextricably associates with total scale number. So, scale number plays an important role to calculate MEV, and the experiments with different scale number will be done in the future to verify the optimized parameters.

\section{Conclusions}

In this paper, an artifact reduction method is proposed based on MEMD and MSE. A new index named MEV is introduced based on MSE to decide the reserved IMFs. All the results mentioned above indicate that our method is effective for artifact reduction. Furthermore, the filtering procedure containing decomposition and reconstruction of signals are absolutely signal-dependent which does not require any user parameter setting, so this article method is fully adaptive and automated for very noisy signals and where artifact level estimation is not possible. The EEG signals can be used to monitor the DOA more correctly after artifact rejection using proposed method. Entropy values based on MSE algorithm have advantages compared to BIS module in the respect of artifacts rejection, and the proposed filtering method in this paper provides a solution to remove the artifacts from EEG signals for monitoring DOA accurately. After regression for range mapping, the results perform better.

\section{Acknowledgments}

This research was financially supported by the Center for Dynamical Biomarkers and Translational Medicine, National Central University, Taiwan which is sponsored by Ministry of Science and Technology (Grant Number: NSC102-2911-I-008-001). Also, it was supported by Chung-Shan Institute of Science \& Technology in Taiwan (Grant Numbers: CSIST-095-V301 and CSIST-095V302) and National Natural Science Foundation of China (Grant Number: 51475342).

\section{Conflict of Interest}

The authors declare no conflict of interest.

\section{Ethical Approval}

This study is approved by Institutional Review Board and written informed consent was also obtained from the permission of the patients. 


\section{References}

[1] L. Vutskits and Z. Xie, "Lasting impact of general anaesthesia on the brain: mechanisms and relevance," Nature Reviews Neuroscience, vol. 17, pp. 705-717, 2016.

[2] G. A. Mashour, "Consciousness UnboundToward a Paradigm of General Anesthesia," The Journal of the American Society of Anesthesiologists, vol. 100, pp. 428-433, 2004.

[3] N. P. Franks, "General anaesthesia: from molecular targets to neuronal pathways of sleep and arousal," Nature Reviews Neuroscience, vol. 9, pp. 370-386, 2008.

[4] E. N. Brown, R. Lydic, and N. D. Schiff, "General anesthesia, sleep, and coma," New England Journal of Medicine, vol. 363, pp. 2638-2650, 2010.

[5] D. A. Kenwright, A. Bernjak, T. Draegni, S. Dzeroski, M. Entwistle, M. Horvat, et al., "The discriminatory value of cardiorespiratory interactions in distinguishing awake from anaesthetised states: a randomised observational study," Anaesthesia, vol. 70, pp. 1356-1368, 2015.

[6] H. Gastaut, "Clinical and electroencephalographical classification of epileptic seizures," Epilepsia, vol. 11, pp. 102-112, 1970.

[7] A. Alvarez, C. Sampedro, M. Laredo, V. Couceiro, P. Perez, R. Cacabelos, et al., "Effects of Cerebrolysin on quantitative EEG activity in elderly controls and in patients with brain damage," European Neuropsychopharmacology, vol. 13, pp. 400-401, Sep 2003.

[8] V. Bajaj and R. B. Pachori, "Automatic classification of sleep stages based on the timefrequency image of EEG signals," Computer Methods and Programs in Biomedicine, vol. 112, pp. 320-328, Dec 2013.

[9] P. Myles, K. Leslie, J. McNeil, A. Forbes, and M. Chan, "Bispectral index monitoring to prevent awareness during anaesthesia: the B-Aware randomised controlled trial," The lancet, vol. 363, pp. 1757-1763, 2004.

[10] P. F. White, J. Tang, G. F. Romero, R. H. Wender, R. Naruse, A. Sloninsky, et al., "A comparison of state and response entropy versus bispectral index values during the perloperative period," Anesthesia and Analgesia, vol. 102, pp. 160-167, Jan 2006.

[11] S. Kreuer, J. Bruhn, R. Larsen, P. Bialas, and W. Wilhelm, "Comparability of Narcotrend (TM) index and bispectral index during propofol anaesthesia," British Journal of Anaesthesia, vol. 93, pp. 235-240, Aug 2004.

[12] G. Kraus, "Mesurement of anesthesia depth, awareness and EEG," Anaesthesist, vol. 50, pp. 950-952, Dec 2001.

[13] T. Stankovski, S. Petkoski, J. Raeder, A. F. Smith, P. V. E. McClintock, and A. Stefanovska, "Alterations in the coupling functions between cortical and cardio-respiratory oscillations due to anaesthesia with propofol and sevoflurane," Phil. Trans. R. Soc. A, vol. 374, p. 20150186, 2016.

[14] T. Stankovski, A. Duggento, P. V. E. McClintock, and A. Stefanovska, "Inference of timeevolving coupled dynamical systems in the presence of noise," Physical Review Letters, vol. 109, p. 024101, 2012.

[15] T. Stankovski, V. Ticcinelli, P. V. E. McClintock, and A. Stefanovska, "Coupling functions in networks of oscillators," New Journal of Physics, vol. 17, p. 035002, 2015.

[16] C. W. Gardiner, Handbook of stochastic methods vol. 3: Springer Berlin, 1985.

[17] J. G. Proakis, Digital signal processing: principles algorithms and applications: Pearson Education India, 2001.

[18] D. L. Donoho, "De-noising by soft-thresholding," Information Theory, IEEE Transactions on, vol. 41, pp. 613-627, 1995.

[19] D. L. Donoho and I. M. Johnstone, "Adapting to unknown smoothness via wavelet shrinkage," Journal of the american statistical association, vol. 90, pp. 1200-1224, 1995.

[20] D. L. Donoho and J. M. Johnstone, "Ideal spatial adaptation by wavelet shrinkage," Biometrika, vol. 81, pp. 425-455, 1994. 
[21] M. Aminghafari, N. Cheze, and J.-M. Poggi, "Multivariate denoising using wavelets and principal component analysis," Computational Statistics \& Data Analysis, vol. 50, pp. 23812398, 2006.

[22] V. Krishnaveni, S. Jayaraman, L. Anitha, and K. Ramadoss, "Removal of ocular artifacts from EEG using adaptive thresholding of wavelet coefficients," Journal of neural engineering, vol. 3, pp. 338-346, 2006.

[23] J. Gao, H. Sultan, J. Hu, and W.-W. Tung, "Denoising nonlinear time series by adaptive filtering and wavelet shrinkage: a comparison," Signal Processing Letters, IEEE, vol. 17, pp. 237-240, 2010.

[24] A. Hyvärinen, J. Karhunen, and E. Oja, Independent component analysis vol. 46: John Wiley \& Sons, 2004.

[25] C. A. Joyce, I. F. Gorodnitsky, and M. Kutas, "Automatic removal of eye movement and blink artifacts from EEG data using blind component separation," Psychophysiology, vol. 41, pp. 313-325, 2004.

[26] D. Iatsenko, P. V. E. McClintock, and A. Stefanovska, "Nonlinear mode decomposition: A noise-robust, adaptive decomposition method," Physical Review E, vol. 92, p. 032916, 2015.

[27] N. E. Huang, Z. Shen, S. R. Long, M. C. Wu, H. H. Shih, Q. Zheng, et al., "The empirical mode decomposition and the Hilbert spectrum for nonlinear and non-stationary time series analysis," Proceedings of the Royal Society of London. Series A: Mathematical, Physical and Engineering Sciences, vol. 454, pp. 903-995, 1998.

[28] N. Rehman and D. P. Mandic, "Multivariate empirical mode decomposition," Proceedings of the Royal Society A: Mathematical, Physical and Engineering Science, vol. 466, pp. 12911302, May 8, 20102010.

[29] D. Safieddine, A. Kachenoura, L. Albera, G. Birot, A. Karfoul, A. Pasnicu, et al., "Removal of muscle artifact from EEG data: comparison between stochastic (ICA and CCA) and deterministic (EMD and wavelet-based) approaches," Eurasip Journal on Advances in Signal Processing, vol. 127, pp. 1-15, 2012.

[30] M. K. I. Molla, M. R. Islam, T. Tanaka, and T. M. Rutkowski, "Artifact suppression from EEG signals using data adaptive time domain filtering," Neurocomputing, vol. 97, pp. 297-308, 2012.

[31] Q. Wei, Q. Liu, S. Z. Fan, C. W. Lu, T. Y. Lin, M. F. Abbod, et al., "Analysis of EEG via Multivariate Empirical Mode Decomposition for Depth of Anesthesia Based on Sample Entropy," Entropy, vol. 15, pp. 3458-3470, Sep 2013.

[32] K. M. Chang, "Arrhythmia ECG Noise Reduction by Ensemble Empirical Mode Decomposition," Sensors, vol. 10, pp. 6063-6080, Jun 2010.

[33] M. Costa, A. L. Goldberger, and C.-K. Peng, "Multiscale entropy analysis of biological signals," Physical Review E, vol. 71, p. 021906, 2005.

[34] M. Costa, A. L. Goldberger, and C.-K. Peng, "Multiscale entropy analysis of complex physiologic time series," Physical review letters, vol. 89, p. 068102, 2002.

[35] J. S. Richman and J. R. Moorman, "Physiological time-series analysis using approximate entropy and sample entropy," American Journal of Physiology-Heart and Circulatory Physiology, vol. 278, pp. 2039-2049, Jun 2000.

[36] G. X. Ouyang, C. Y. Dang, and X. L. Li, "Multiscale Entropy Analysis of Eeg Recordings in Epileptic Rats," Biomedical Engineering-Applications Basis Communications, vol. 21, pp. 169176, Jun 2009.

[37] G. Deco, V. K. Jirsa, and A. R. McIntosh, "Emerging concepts for the dynamical organization of resting-state activity in the brain," Nature Reviews Neuroscience, vol. 12, pp. 43-56, 2011.

[38] G. M. Friesen, T. C. Jannett, M. A. Jadallah, S. L. Yates, S. R. Quint, and H. T. Nagle, "A comparison of the noise sensitivity of nine QRS detection algorithms," Biomedical Engineering, IEEE Transactions on, vol. 37, pp. 85-98, 1990.

[39] C. Brunner, R. Leeb, G. R. Muller-Putz, A. Schlogl, and G. Pfurstscheller. (2008). BCI Competition 2008 - Graz data set A. Available: http://www.bbci.de/competition/iv/desc_2a.pdf 
[40] R. A. Harvey, M. Mycek, and P. Champe, "Lippincott's Illustrated Reviews: Pharmacology," Trends in Pharmacological Sciences, vol. 19, p. 38, 1998.

[41] J. Schüttler and H. Schwilden, Modern anesthetics: Springer.

[42] H. S. Joo and W. J. Perks, "Sevoflurane versus propofol for anesthetic induction: a metaanalysis," Anesthesia \& Analgesia, vol. 91, pp. 213-219, 2000.

[43] G. Kumar, C. Stendall, R. Mistry, K. Gurusamy, and D. Walker, "A comparison of total intravenous anaesthesia using propofol with sevoflurane or desflurane in ambulatory surgery: systematic review and meta-analysis," Anaesthesia, vol. 69, pp. 1138-1150, 2014.

[44] Q. Liu, Y.-F. Chen, S.-Z. Fan, M. F. Abbod, and J.-S. Shieh, "EEG signals analysis using multiscale entropy for depth of anesthesia monitoring during surgery through artificial neural networks," Computational and mathematical methods in medicine, vol. 2015, 2015.

[45] P. Flandrin, G. Rilling, and P. Goncalves, "Empirical mode decomposition as a filter bank," Signal Processing Letters, IEEE, vol. 11, pp. 112-114, 2004.

[46] N. ur Rehman and D. P. Mandic, "Filter Bank Property of Multivariate Empirical Mode Decomposition," Signal Processing, IEEE Transactions on, vol. 59, pp. 2421-2426, 2011.

[47] Z. Wu and N. E. Huang, "A study of the characteristics of white noise using the empirical mode decomposition method," Proceedings of the Royal Society of London. Series A: Mathematical, Physical and Engineering Sciences, vol. 460, pp. 1597-1611, 2004.

[48] A.-O. Boudraa and J.-C. Cexus, "EMD-based signal filtering," Instrumentation and Measurement, IEEE Transactions on, vol. 56, pp. 2196-2202, 2007.

[49] A. Boudraa, J. Cexus, and Z. Saidi, "EMD-based signal noise reduction," International Journal of Signal Processing, vol. 1, pp. 33-37, 2004.

[50] D. E. Lake, J. S. Richman, M. P. Griffin, and J. R. Moorman, "Sample entropy analysis of neonatal heart rate variability," American Journal of Physiology-Regulatory Integrative and Comparative Physiology, vol. 283, pp. 789-797, Sep 2002.

[51] R. Shalbaf, H. Behnam, J. Sleigh, and L. Voss, "Measuring the effects of sevoflurane on electroencephalogram using sample entropy," Acta Anaesthesiologica Scandinavica, vol. 56, pp. 880-889, Aug 2012.

[52] H. Aurlien, I. O. Gjerde, J. H. Aarseth, G. Eldoen, B. Karlsen, H. Skeidsvoll, et al., "EEG background activity described by a large computerized database," Clinical Neurophysiology, vol. 115, pp. 665-673, Mar 2004.

[53] G. Tsolis and T. D. Xenos, "Signal denoising using empirical mode decomposition and higher order statistics," Int J Signal Proc Image Proc Pattern Recog, vol. 4, pp. 91-106, 2011.

[54] Y. Kopsinis and S. McLaughlin, "Development of EMD-based denoising methods inspired by wavelet thresholding," Signal Processing, IEEE Transactions on, vol. 57, pp. 1351-1362, 2009. 


\section{Figure Legends}

Fig. 1 Quasi-uncorrupted EEG signal.

Fig. 2 EEG corrupted with artifacts: (a) EOG; (b) EMG; (c) power-line; (d) baseline drift.

Fig. 3 Noisy EEG signals collected during surgery: (a) EOG; (b) EMG; (c) baseline drift due to the movement of patient.

Fig. 4 The distribution of MSE of one case's EEG signal corrupted with different artifacts: (a) EOG; (b) EMG; (c) power-line; (d) baseline drift. The level of each artifact increases with signal to noise ratio from $10 \mathrm{~dB}$ to $-20 \mathrm{~dB}$ with step $-1 \mathrm{~dB}$, and the total number of scale factor is 20. Moreover, the distribution of MSE of the original EEG signal is shown without artifact in the bottom of figures.

Fig. 5 MEV analysis of EEG corrupted with different artifacts: (a) EOG; (b) EMG; (c) power-line; (d) baseline drift. The points on the left of dotted line represent MEV when there is no artifact in EEG signal.

Fig. 6 SNR difference between after and before denoising with respect to the SNR before denoising in the cases of EEG corrupted with different artifacts: (a) EOG; (b) EMG; (c) power-line; (d) baseline drift.(a) shows the plots of the SNR difference between after and before de-noising versus the SNR level before de-noising for the EEG signals corrupted with EOG artifact. The improvements in the SNR increase progressively with input SNR level except individual points. It almost increases linearly with the increasing of input SNR. (b), (c) and (d) show the plots of results for EEG corrupted with EMG, power-line and baseline drift respectively.

Fig. 7 Illustration of the relationship between entropy values and BIS in monitoring DOA during the whole operation. (a) the original EEG recordings; (b) the filtered EEG recordings; (c) the real time BIS collected by MP60 and entropy after regression during whole surgery; (d) the MSE results calculated from EEG recordings when scale is 1 before and after filtering through our proposed method. The entropy after filtering and regression is very close to BIS in (c). It is evident that the EEG signal is very noisy so that entropy values are inaccurate at pre-operation and recovery stages before filtering in (d), however, the corresponding values is more reasonable after filtering.

Fig. 8 Entropy values and BIS in monitoring DOA in strong artifact background. (a) Original EEG signal; (b) The corresponding entropy and BIS. Both entropy and BIS are very low (BIS = -1) in position (1), because the poor contact causes EEG recording to fail occasionally. They both cannot monitor the DOA correctly under the circumstances. The signal is disturbed by artifact seriously in the positions marked with (2), (3) and (4), during the pre-operation and the initial stage of induction, patients have high levels of consciousness, and they blink frequently, so EEG is seriously contaminated by EOG artifacts, BIS = -1 but entropy values after filtering and regression are correct, such as (2) and (3), and in (4), duo to the using of electrical knife, the original signal is also disturbed seriously, the BIS values are -1 , but the entropy values are normal. 


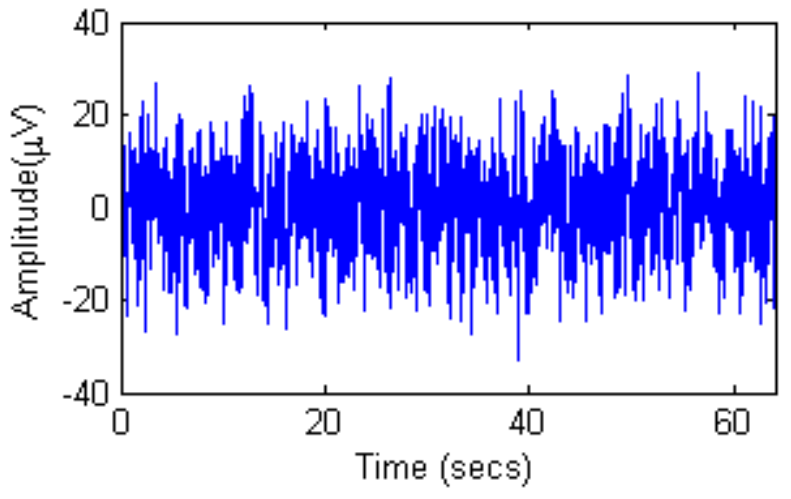

Fig 1. 

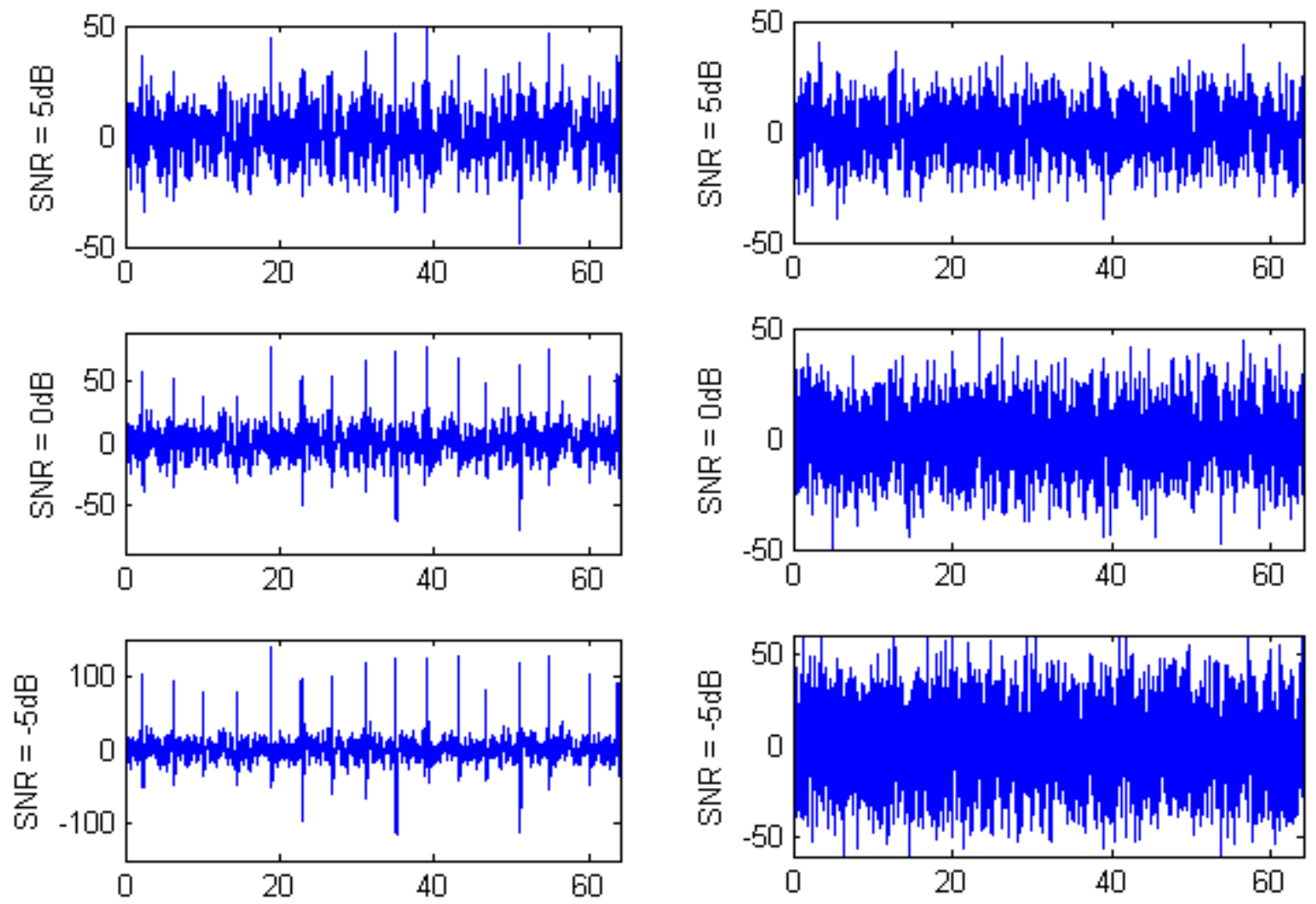

(a)

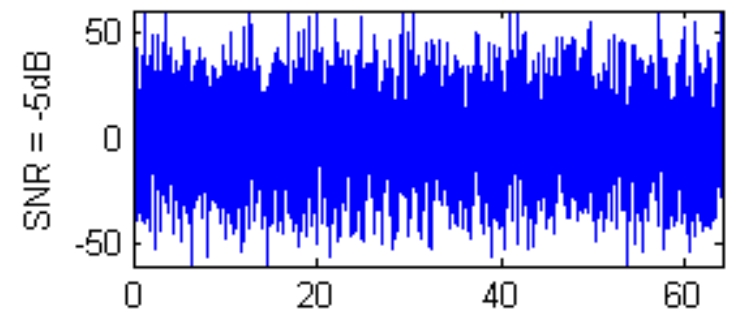

(b)
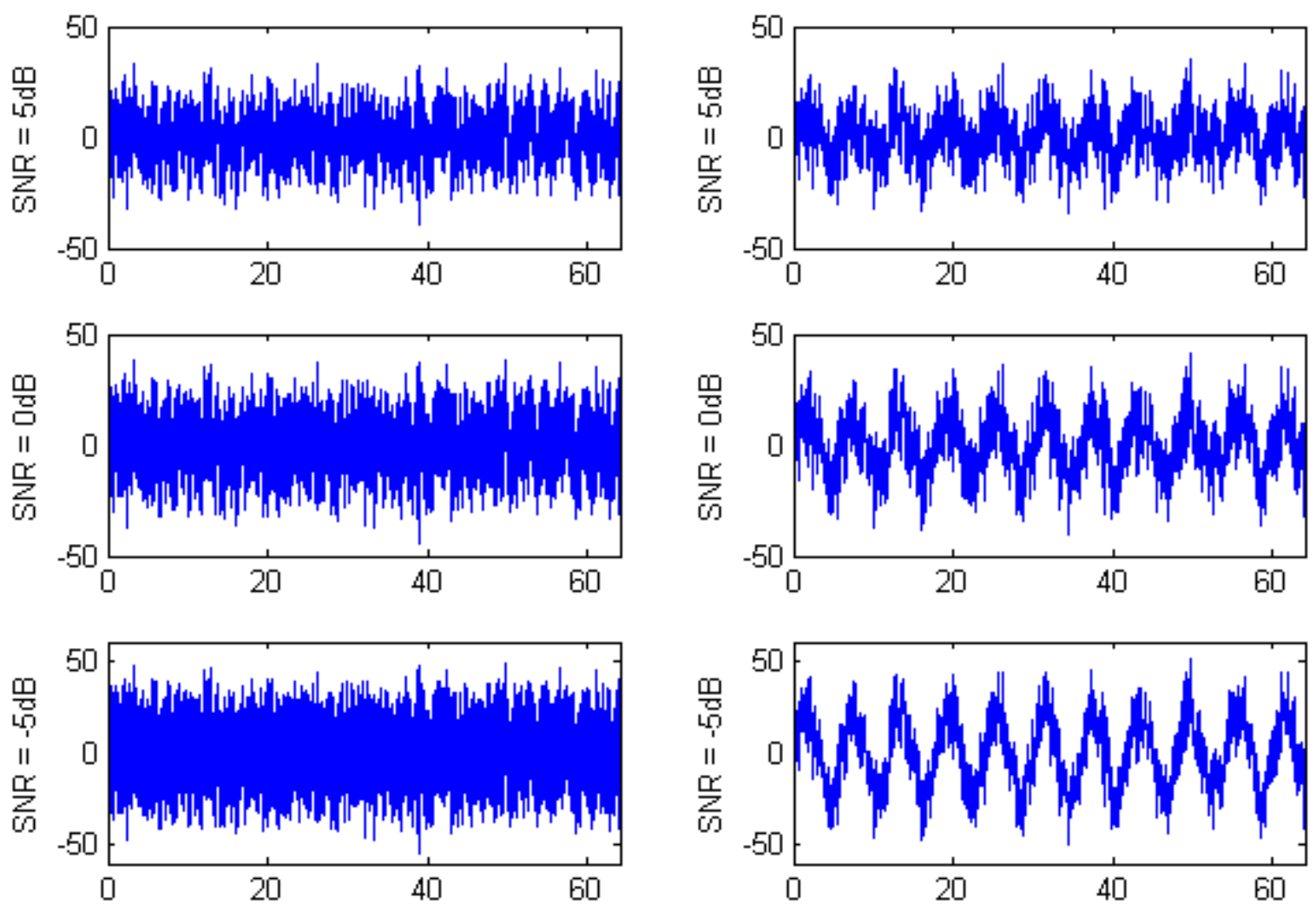

(c)

(d)

Fig 2. 


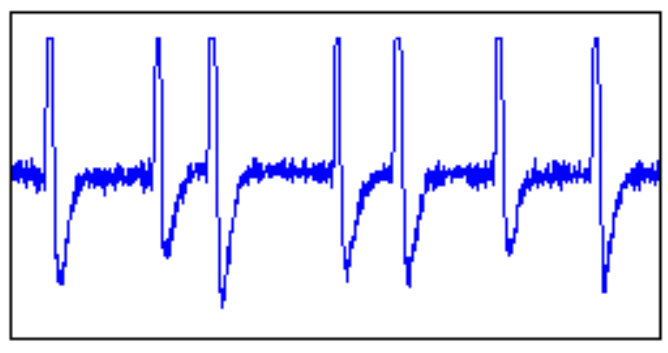

(a)

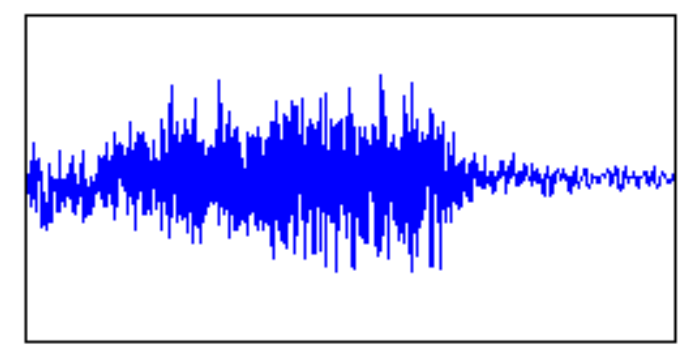

(b)

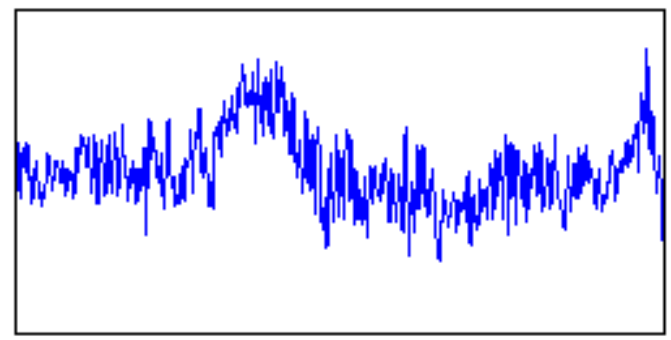

(c)

Fig 3. 

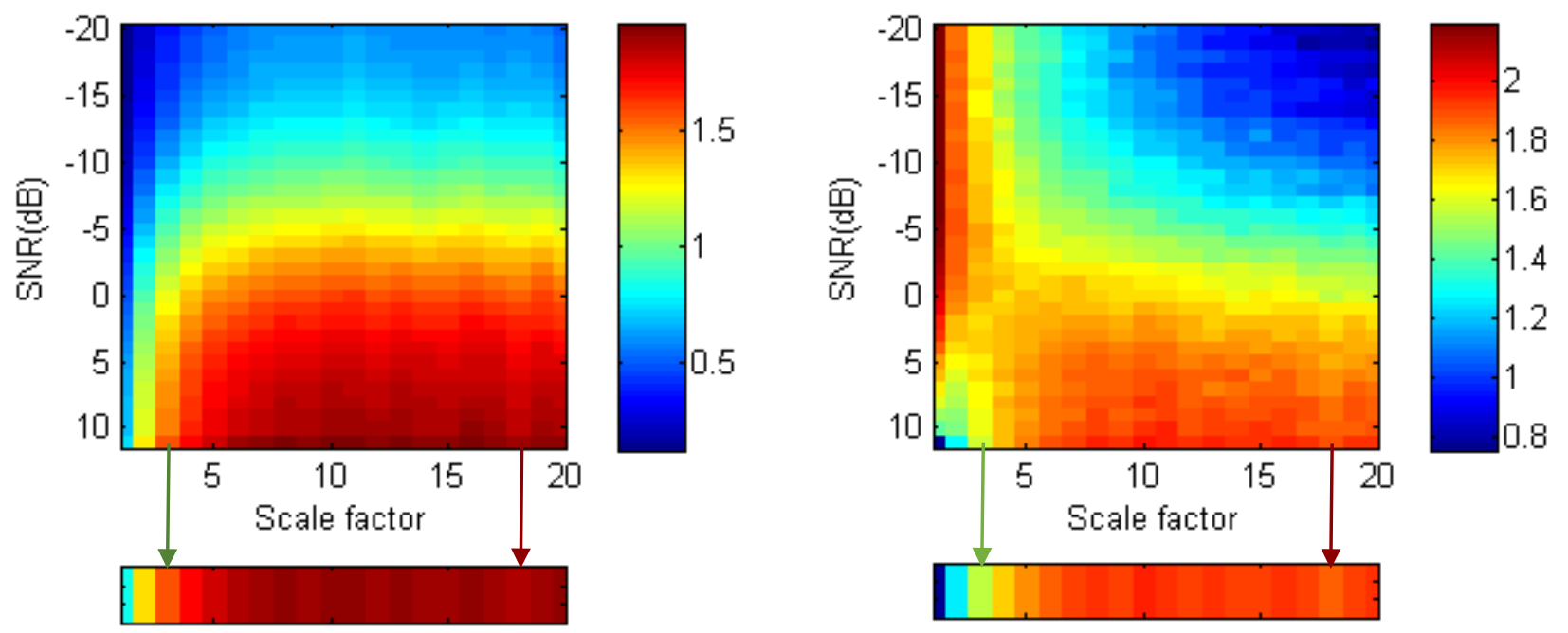

(a)

(b)
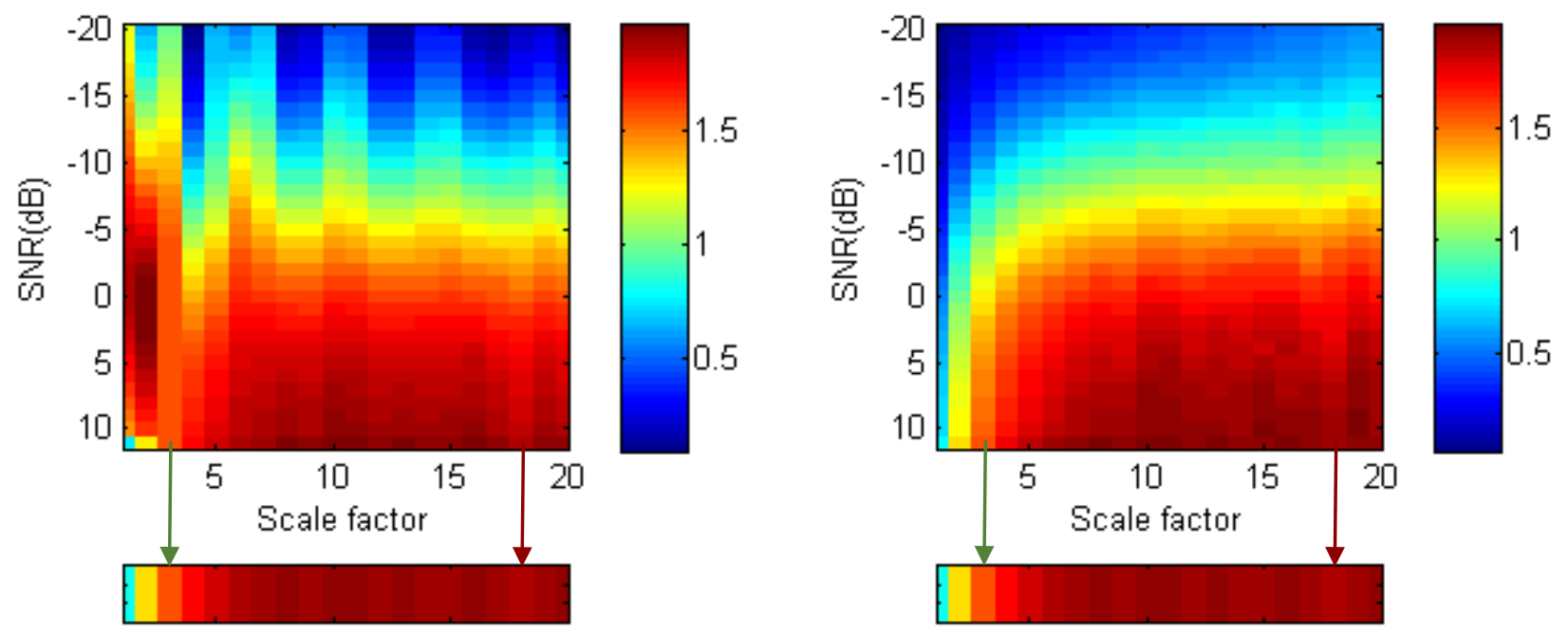

(c)

(d)

Fig 4. 


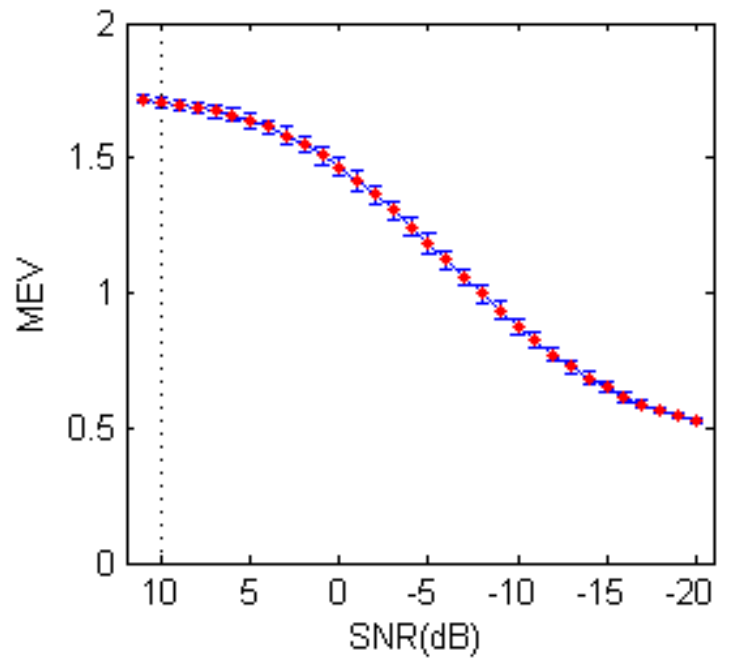

(a)

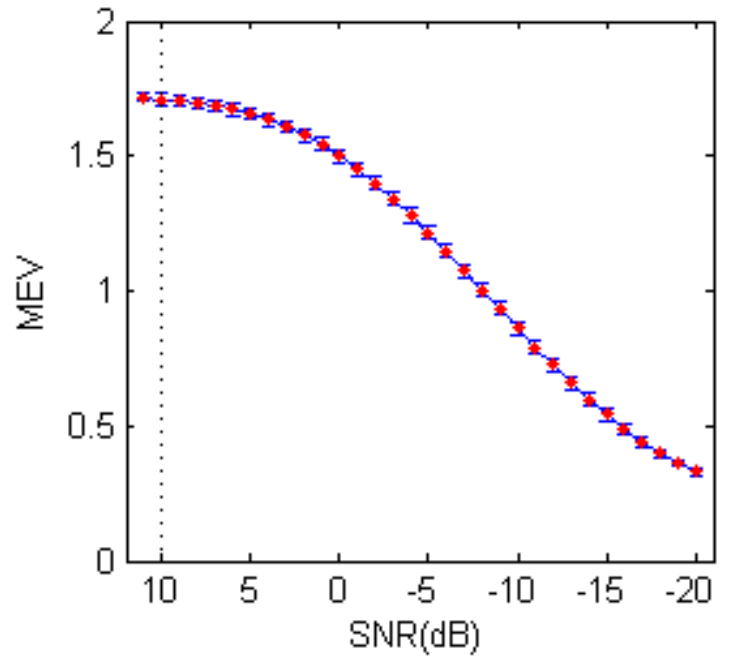

(c)



(b)

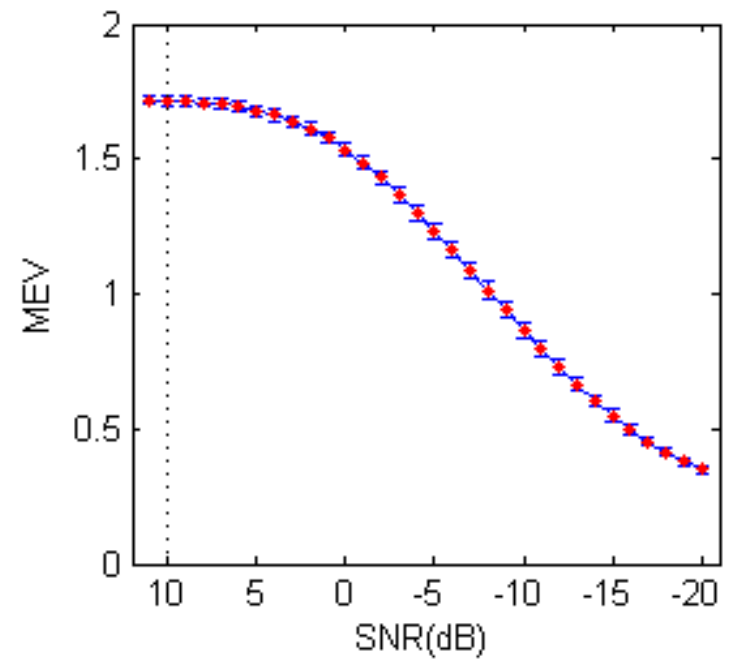

(d)

Fig 5. 


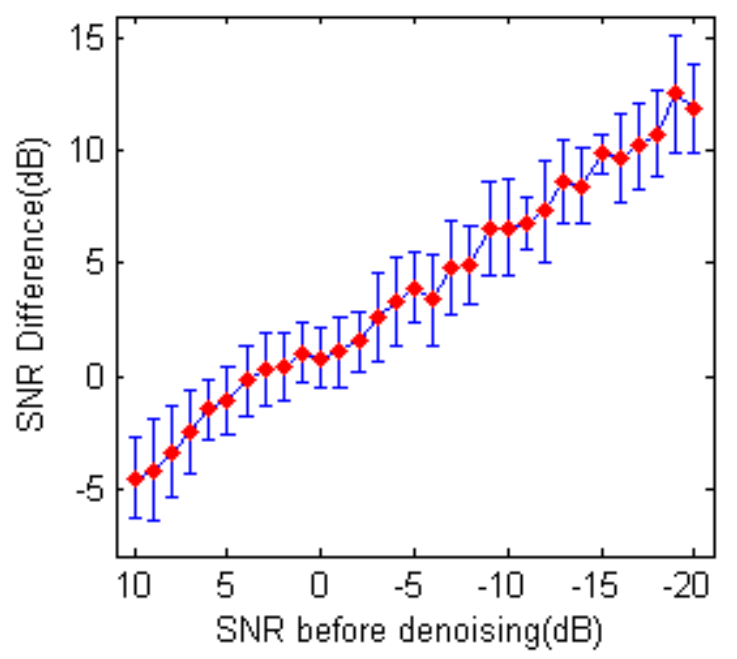

(a)

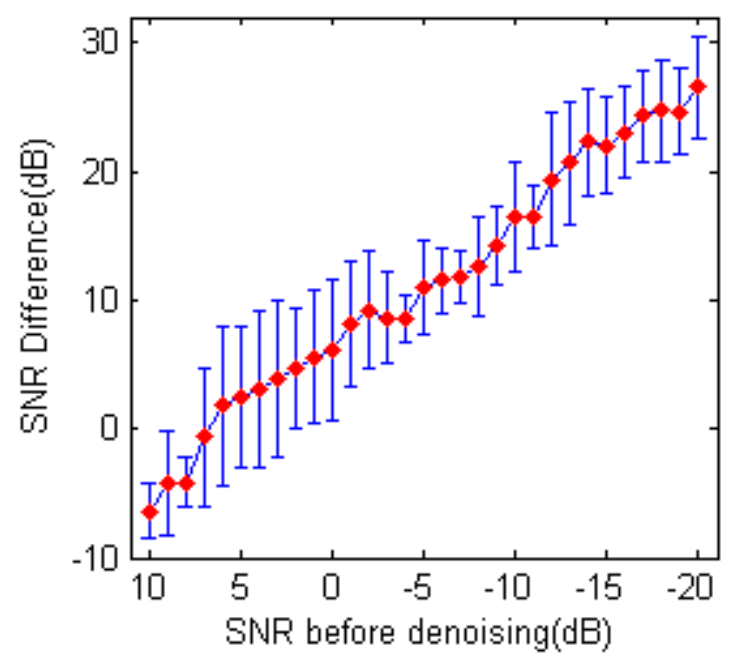

(c)



(b)

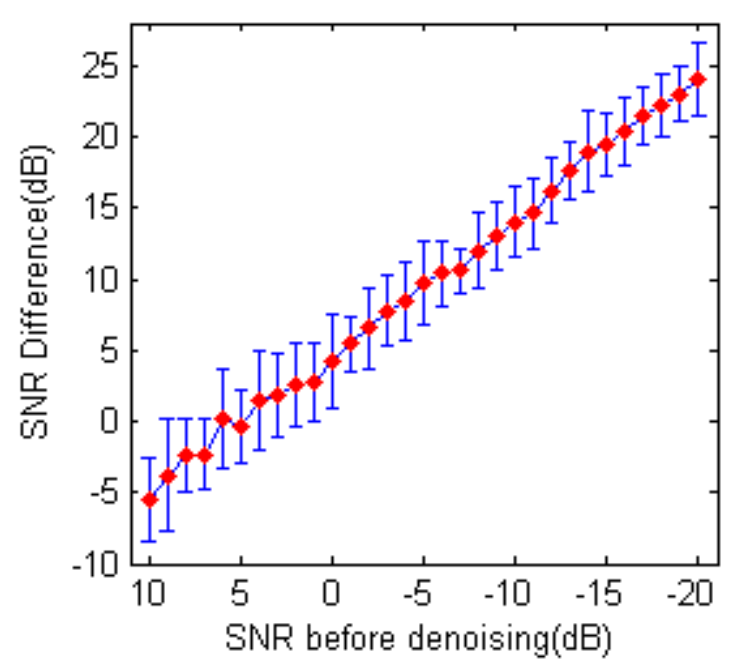

(d)

Fig 6. 


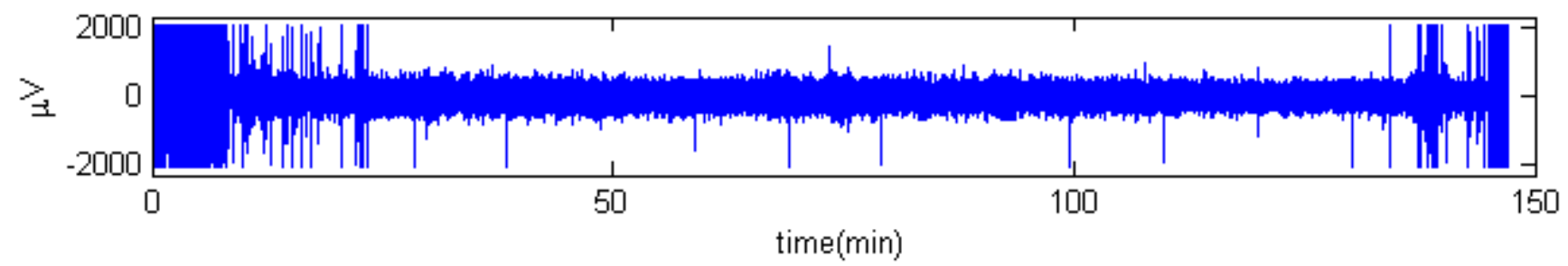

(a)

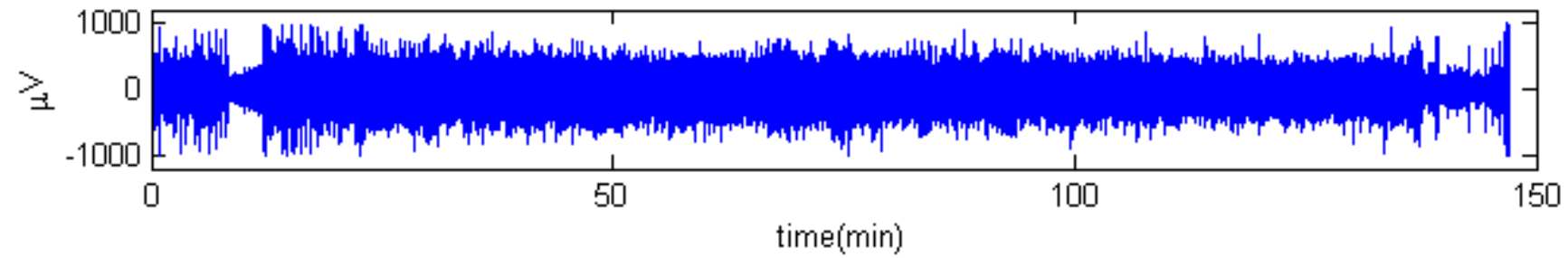

(b)

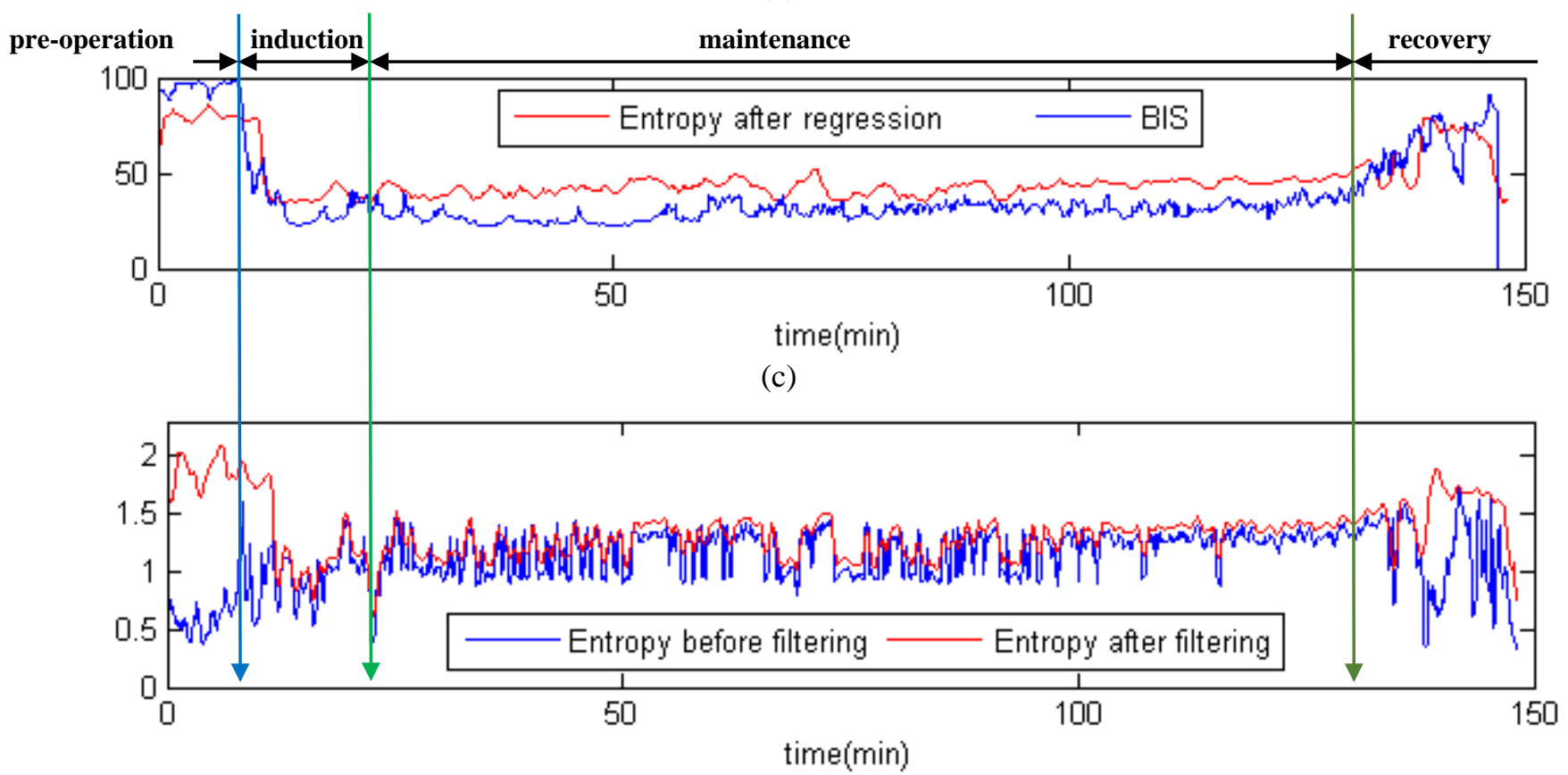

(d)

Fig 7. 


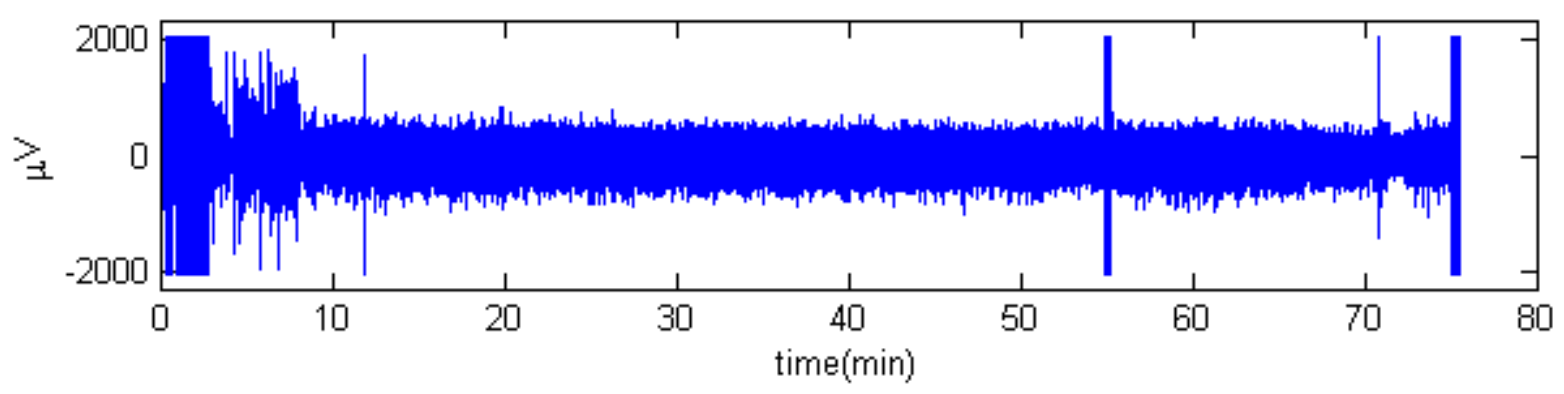

(a)

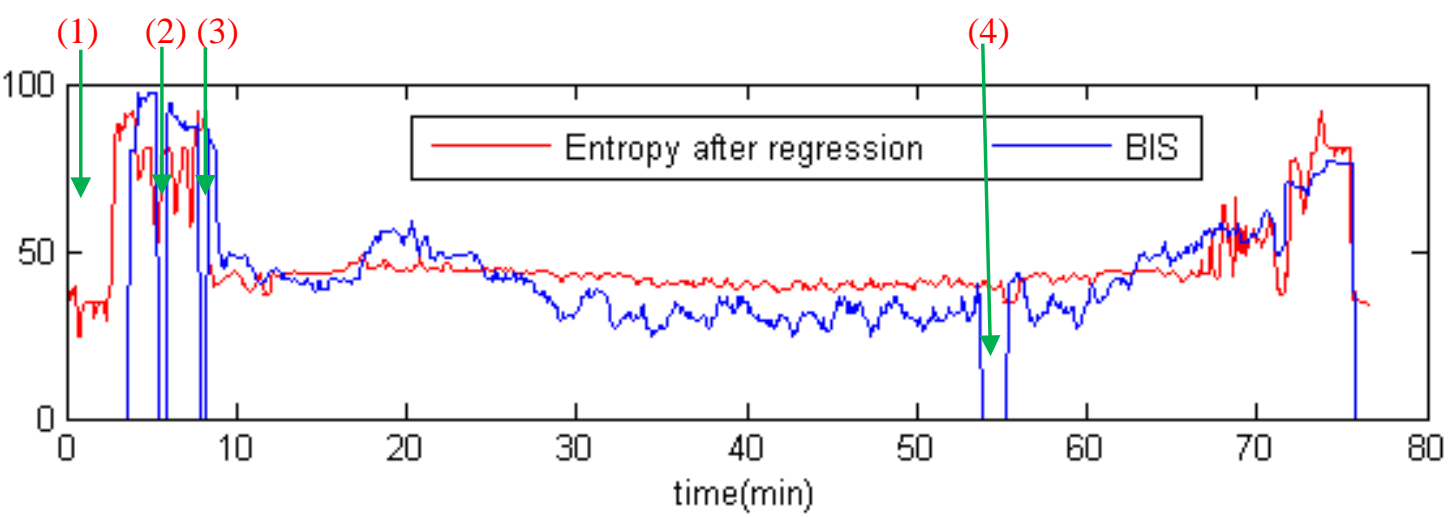

(b)

Fig 8. 
Table 1. Performance improvement of the proposed method corresponding to different artifact intensity.

\begin{tabular}{|c|c|c|c|c|}
\hline \multirow{2}{*}{$\begin{array}{c}\text { SNR } \\
\text { before(dB) }\end{array}$} & \multicolumn{4}{|c|}{ SNR improvement between after and before de-noising (dB) } \\
\hline & EOG & EMG & Power-line & Baseline drift \\
\hline 10 & $-4.54 \pm 1.78$ & $-6.21 \pm 1.84$ & $-6.37 \pm 2.09$ & $-5.53 \pm 2.91$ \\
\hline 9 & $-4.18 \pm 2.23$ & $-5.31 \pm 1.92$ & $-4.15 \pm 4.05$ & $-3.78 \pm 3.90$ \\
\hline 8 & $-3.37 \pm 2.06$ & $-3.50 \pm 2.57$ & $-4.14 \pm 1.89$ & $-2.42 \pm 2.56$ \\
\hline 7 & $-2.47 \pm 1.83$ & $-3.35 \pm 2.15$ & $-0.58 \pm 5.37$ & $-2.30 \pm 2.45$ \\
\hline 6 & $-1.50 \pm 1.34$ & $-2.14 \pm 1.90$ & $1.82 \pm 6.15$ & $0.21 \pm 3.52$ \\
\hline 5 & $-1.12 \pm 1.54$ & $-0.05 \pm 2.42$ & $2.41 \pm 5.48$ & $-0.40 \pm 2.59$ \\
\hline 4 & $-0.23 \pm 1.53$ & $-0.27 \pm 2.17$ & $3.06 \pm 6.15$ & $1.50 \pm 3.49$ \\
\hline 3 & $0.27 \pm 1.63$ & $0.26 \pm 1.27$ & $3.92 \pm 6.11$ & $1.88 \pm 2.95$ \\
\hline 2 & $0.36 \pm 1.49$ & $1.67 \pm 1.64$ & $4.74 \pm 4.61$ & $2.56 \pm 2.91$ \\
\hline 1 & $1.00 \pm 1.32$ & $1.92 \pm 1.48$ & $5.61 \pm 5.14$ & $2.80 \pm 2.71$ \\
\hline 0 & $0.80 \pm 1.37$ & $2.53 \pm 0.96$ & $6.08 \pm 5.51$ & $4.21 \pm 3.35$ \\
\hline-1 & $1.06 \pm 1.53$ & $3.55 \pm 0.75$ & $8.16 \pm 4.97$ & $5.49 \pm 1.93$ \\
\hline-2 & $1.54 \pm 1.35$ & $4.00 \pm 1.06$ & $9.21 \pm 4.60$ & $6.55 \pm 2.81$ \\
\hline-3 & $2.65 \pm 1.98$ & $4.60 \pm 0.63$ & $8.66 \pm 3.51$ & $7.80 \pm 2.50$ \\
\hline-4 & $3.27 \pm 1.97$ & $5.65 \pm 0.63$ & $8.59 \pm 1.84$ & $8.50 \pm 2.78$ \\
\hline-5 & $3.94 \pm 1.62$ & $5.92 \pm 0.78$ & $11.02 \pm 3.58$ & $9.74 \pm 2.96$ \\
\hline-6 & $3.38 \pm 2.01$ & $6.78 \pm 0.50$ & $11.56 \pm 2.54$ & $10.42 \pm 2.29$ \\
\hline-7 & $4.83 \pm 2.10$ & $7.45 \pm 0.46$ & $11.82 \pm 1.97$ & $10.59 \pm 1.61$ \\
\hline-8 & $4.95 \pm 1.73$ & $7.49 \pm 0.59$ & $12.63 \pm 3.94$ & $12.02 \pm 2.74$ \\
\hline-9 & $6.53 \pm 2.11$ & $7.80 \pm 0.73$ & $14.28 \pm 2.98$ & $13.01 \pm 2.40$ \\
\hline-10 & $6.59 \pm 2.17$ & $8.27 \pm 0.84$ & $16.50 \pm 4.30$ & $14.05 \pm 2.52$ \\
\hline-11 & $6.79 \pm 1.14$ & $8.78 \pm 0.99$ & $16.52 \pm 2.49$ & $14.60 \pm 2.39$ \\
\hline-12 & $7.34 \pm 2.27$ & $8.76 \pm 0.83$ & $19.36 \pm 5.14$ & $16.23 \pm 2.24$ \\
\hline-13 & $8.65 \pm 1.85$ & $8.70 \pm 1.31$ & $20.68 \pm 4.74$ & $17.72 \pm 2.02$ \\
\hline-14 & $8.43 \pm 1.66$ & $9.54 \pm 0.96$ & $22.29 \pm 4.12$ & $18.98 \pm 2.78$ \\
\hline-15 & $9.89 \pm 0.86$ & $9.07 \pm 1.20$ & $22.04 \pm 3.71$ & $19.47 \pm 2.18$ \\
\hline-16 & $9.65 \pm 1.98$ & $9.67 \pm 1.12$ & $23.03 \pm 3.56$ & $20.40 \pm 2.41$ \\
\hline-17 & $10.22 \pm 1.94$ & $9.56 \pm 1.06$ & $24.33 \pm 3.61$ & $21.54 \pm 2.05$ \\
\hline-18 & $10.74 \pm 1.92$ & $10.00 \pm 1.48$ & $24.75 \pm 3.91$ & $22.23 \pm 2.14$ \\
\hline-19 & $12.56 \pm 2.62$ & $9.59 \pm 1.51$ & $24.63 \pm 3.34$ & $23.04 \pm 1.86$ \\
\hline-20 & $11.90 \pm 1.97$ & $9.38 \pm 1.34$ & $26.53 \pm 3.89$ & $24.08 \pm 2.51$ \\
\hline mean \pm std & $3.87 \pm 4.95$ & $4.62 \pm 4.99$ & $11.26 \pm 9.50$ & $9.52 \pm 8.81$ \\
\hline
\end{tabular}


Table 2. The statistical analysis results between entropy and BIS during each of the four stages. The results are expressed as mean \pm standard deviation. The test of Pearson's correlation is performed between entropy values measured from original and reconstructed EEG signals and BIS in the twenty one patients during each stage respectively.

\begin{tabular}{cccccc}
\hline \hline \multirow{2}{*}{ Stages } & \multicolumn{2}{c}{ before filtering } & \multicolumn{2}{c}{ after filtering } & BIS \\
\cline { 2 - 6 } & mean \pm std & $\begin{array}{c}\text { correlation } \\
(\text { Entropy vs. BIS)* }\end{array}$ & mean \pm std & $\begin{array}{c}\text { correlation } \\
(\text { Entropy vs. BIS)* }\end{array}$ & mean \pm std \\
\hline Pre-operation & $0.85 \pm 0.26$ & $0.18(\mathrm{p}=0.434)$ & $1.65 \pm 0.20$ & $0.77(\mathrm{p}<0.001)$ & $84.29 \pm 14.49$ \\
Induction & $1.00 \pm 0.14$ & $0.43(\mathrm{p}=0.050)$ & $1.33 \pm 0.31$ & $0.80(\mathrm{p}<0.001)$ & $62.91 \pm 11.68$ \\
Maintenance & $1.14 \pm 0.13$ & $0.74(\mathrm{p}<0.001)$ & $1.14 \pm 0.13$ & $0.73(\mathrm{p}<0.001)$ & $41.18 \pm 7.26$ \\
Recovery & $1.23 \pm 0.30$ & $0.45(\mathrm{p}=0.041)$ & $1.39 \pm 0.25$ & $0.77(\mathrm{p}<0.001)$ & $62.66 \pm 10.80$ \\
\hline \hline
\end{tabular}

*. Correlation is significant at the 0.01 level (2-tailed). If the significance level $\mathrm{p}$ is less than 0.01 then the correlation is statistical significant and the two variables have a linear relationship.

Table 3. The statistical analysis results between entropy and BIS during the whole operation in twenty one surgeries. The wavelet denoising method and our proposed method are used to filter the original signal, respectively.

\begin{tabular}{cccccc}
\hline \hline \multirow{2}{*}{ Case } & before filtering & \multicolumn{2}{c}{ after filtering } & \multicolumn{3}{c}{ ANN } \\
& & Wavelet & MEMD_MSE & Wavelet & MEMD_MSE \\
\hline 1 & 0.57 & 0.66 & 0.84 & 0.78 & 0.91 \\
2 & 0.04 & 0.04 & 0.60 & 0.26 & 0.71 \\
3 & -0.29 & 0.10 & 0.80 & 0.33 & 0.92 \\
4 & -0.39 & -0.07 & 0.67 & 0.48 & 0.86 \\
5 & 0.12 & 0.21 & 0.54 & 0.31 & 0.68 \\
6 & 0.39 & 0.34 & 0.65 & 0.42 & 0.79 \\
7 & 0.34 & 0.47 & 0.66 & 0.30 & 0.73 \\
8 & 0.44 & -0.08 & 0.64 & 0.29 & 0.77 \\
9 & -0.34 & -0.36 & 0.58 & -0.14 & 0.71 \\
10 & 0.41 & 0.50 & 0.67 & 0.58 & 0.84 \\
11 & -0.03 & 0.18 & 0.61 & 0.49 & 0.75 \\
12 & 0.52 & 0.15 & 0.71 & 0.41 & 0.76 \\
13 & 0.27 & 0.20 & 0.59 & 0.30 & 0.78 \\
14 & -0.11 & 0.33 & 0.56 & 0.32 & 0.69 \\
15 & 0.31 & 0.19 & 0.74 & 0.15 & 0.84 \\
16 & 0.05 & 0.12 & 0.57 & 0.50 & 0.74 \\
17 & 0.36 & 0.39 & 0.64 & 0.39 & 0.74 \\
18 & -0.31 & -0.31 & 0.51 & 0.25 & 0.67 \\
19 & 0.53 & 0.41 & 0.58 & 0.21 & 0.63 \\
20 & 0.18 & -0.08 & 0.53 & 0.44 & 0.65 \\
21 & -0.10 & -0.20 & 0.47 & 0.37 & 0.65 \\
mean \pm std & $0.14 \pm 0.30$ & $0.15 \pm 0.27$ & $0.63 \pm 0.09$ & $0.35 \pm 0.18$ & $0.75 \pm 0.08$ \\
\hline \hline
\end{tabular}

